\title{
Acute and Subacute Oral Toxicity Evaluation of Commiphora Campestris Methanolic Stem Bark Extract (CASBMCE) In Mice and Rats
}

\author{
Furaha Nyunza, Edna Makule and Musa Chacha* \\ School of Life Sciences, Nelson Mandela African Institution of Science and Technology, Tanzania
}

Submission: July 31, 2017; Published: November 16, 2017

*Corresponding author: Musa Chacha, School of Life Sciences, Nelson Mandela African Institution of Science and Technology, Tanzania, Tel: +255753458177; Email: musa.chacha@nm-aist.ac.tz

\begin{abstract}
This study was done to evaluate the acute and sub acute toxicity of Camiphora campestris Methanolic Stem Bark Extract (CASBMCE). In acute toxicity tests four groups of mice ( $\mathrm{n}=5 / \mathrm{group} / \mathrm{sex}$ ) were orally treated with doses of 300,600 and $1200 \mathrm{mg} / \mathrm{kg}$ and clinical manifestation, body weight and mortality was recorded for 14 days. In sub acute toxicity study rats received CASBMCE by gavages' at doses $150 \mathrm{mg} / \mathrm{kg}, 200 \mathrm{mg} / \mathrm{kg}$ and $250 \mathrm{mg} / \mathrm{kg}$ ( $\mathrm{n}=5$ rats/group/sex) for 28 days and body weight, relative organ weight, biochemical, hematological and histopathological changes in vital organs were determined. In acute toxicity study mice showed signs of behavioral changes and resumed to their normal bright status. Death was recorded at a dose of 600 and $1200 \mathrm{mg} / \mathrm{kg}$. In sub acute tests no mortality was recorded animals were bright and normal. CASBMCE increased the levels of the liver enzymes (AST \& ALT) with increasing doses. Biochemical examination showed differences between CASBMCE tested and control group. Hematology showed significant differences in CASBMCE tested parameters compared to control group. CASBMCE showed toxicity in the body tissues, in high sub acute doses. This study concludes that acute toxicity study CASBMCE, produced mortality in males and females at a dose of $600 \mathrm{mg} / \mathrm{kg}$ and $1200 \mathrm{mg} / \mathrm{kg}$. In sub acute doses CASBMCE, produced no mortality. Major effects were rise of glucose levels, decreased vital organs size, and thickened female lung alveolar walls that probably increased RBC counts. CASBMCE reduced cholesterol levels indicating antilipidemic effect. More research to validate safety doses in humans is still needed..
\end{abstract}

Keywords: Commiphora campestris; Acute toxicity; Sub acute toxicity; Dimethyl sulphoxide (DSMO)

Abbreviations: TFDA: Tanzania Food and Drugs Authority; SUA: Sokoine University of Agriculture; WBCC: White Blood Cell Count; RBCC: Red Blood Cell Count; CASBMCE: Commiphora campestris Methanolic Stem Bark Extract

\section{Introduction}

Commiphora campestris stem barks are used by the Pare tribe for treatment of several ailments such as diarrhea, coughs and wounds. Therefore, this study aimed on evaluating toxicity profile of CASBMCE using acute toxicity study in mice and subacute study in a rat model. Medicinal plants prepared using African knowledge have played a significant role in provision of health care services for many years even before the introduction of orthodox conventional medicines in African countries. The number of herbal products imported in the country has also increased in recent years. For instance in 2005, 2006 and 2014, Tanzania Food and Drug Authority (TFDA) registered 13, 13 and 8 herbal products in Tanzania, respectively $[1,2]$. The growing demand for herbal products necessitates the provision of evidence on the efficacy, safety and quality of herbal medicines [3].
It is in this vein that medicinal plants used for management of microbial infections and non infectious diseases in Tanzania are being evaluated for their antimicrobial and efficacy properties by Natural Products Research (NPR) in Tanzania. One of such plants is Commiphora campestris also called "Msighe" in the Pare language; it has been used for many years by Pare communities for the management of coughs, wounds, diarrhoea, gastrointestinal infections and has recently found its way to the markets. Godfrey [4] established that the latex from the chopped stem bark inhibits the growth of Klebsiella oxytoca (clinical isolate), Klebsiella pneumoniae (ATCC700603), Salmonella kisarawe (clinical isolate), Proteus mirabilis (NCTC 1075), Salmonella typhi (NCTC 8385), Pseudomonas aeruginosa (ATCC 29953), Escherichia coli (ATCC 25922), Cryptococcus neuformans (clinical isolate) and Candida albicans (ATCC90028). 
It is therefore likely $C$. campestris is a potential source of drug templates for the management of Gram negative bacteria and fungi. This discovery necessitated toxicological studies on the $C$. campestris [5].

\section{Methods and Material}

\section{Plant materials}

Commiphora campestris stem barks were collected from Pare Mountains at Kisiwani Village in Same District, Kilimanjaro, Tanzania in April, 2016. Identification was performed by Mr. Emmanuel Mboya, a botanist from Tropical Pesticides Research Institute (TPRI), Arusha, Tanzania. Voucher specimens were stored at NM-AIST.

\section{Preparation of the CASBMCE}

Fresh collected Commiphora campestris barks were washed and dried under the shade, then pulverized into powder using Swinging Traditional Chinese Medicine Pulveriser, Diaxiang Electronic Equipment (DXF-20D). 500g of the powder was consecutively extracted in three solvents namely chloroform, ethyl acetate and methanol, by soaking in 1 litre of a solvent for 72 hours then sieved and filtered using Whatman filter size 40. After extraction crude extracts were evaporated using a rotary vacuum evaporator at $42{ }^{\circ} \mathrm{C}$, weighed and stored at $4{ }^{\circ} \mathrm{C}$ until used.

\section{Animals and treatment}

In acute toxicity study, mature forty (40) white Swiss Albino mice (20 females and 20 males) weighing between 19-28g were divided into four groups of five mice each and in sub acute toxicity study, forty (40) Winstar albino rats (20 females and 20 males) weighing 62-96g for male sex and 45-77g for female sex, bought from the Sokoine University of Agriculture (SUA) were used. Same sex and litter were kept in a meshed cage size $10 \times 10 \times 10 \mathrm{~cm}$ covered with saw dust beddings to avoid mating and fighting. Light was set at 12 hour light supply and 12 hour darkness. Each cage was identified for extract and dose level. Animals were left to acclimatize for 1 week before administration with CASBMCE. The ethical clearance number NIMR/HQ/R.8a/Vol.IX/2396 was sought from NIMR Tanzania.

\section{Acute toxicity evaluation}

Acute toxicity study was done according to EOCD method. Mice were administered single oral dose of CASBMCE at 300 , 600 and $1200 \mathrm{mg} / \mathrm{kg}$ while control group received $10 \%$ DMSO as extract vehicle. After administration, all test groups were observed for fourteen (14) days for ill health. Mice were marked with one tail mark for $300 \mathrm{mg} / \mathrm{kg}$, two tail marks for $600 \mathrm{mg} /$ $\mathrm{kg}$ and three tail marks for $1200 \mathrm{mg} / \mathrm{kg}$ and control group was unmarked. The mice were fed and water drunken ad-libitum. The method of Lorke [6] was adopted for calculating the lethal dose fifty using the formula below

$L D 50=\sqrt{\mathrm{Dhl}} * \mathrm{~lm}$

\section{Where}

a. Dhl is the highest dose without mortality.

b. Dlm is the lowest dose with mortality.

c. LD50 is the Lethal Dose 50.

\section{Subacute toxicity evaluation}

Sub acute toxicity was carried out according to EOCD [7]. Forty (40) rats were divided into two groups per sex (20 male rats and 20 female rats). 20 rats per each sex were grouped into four groups of five rats per group. Males and females were separated to avoid mating and fighting. The male rats were grouped such that, the first group was a control group and received $10 \%$ DMSO, the second group received a dose of $150 \mathrm{mg} / \mathrm{kg}$, third group received $200 \mathrm{mg} / \mathrm{kg}$ and the fourth group received $250 \mathrm{mg} /$ $\mathrm{kg}$ of CASBMCE. The same grouping was done to female sex rats. Three dose categories of $(150 \mathrm{mg} / \mathrm{kg}, 200 \mathrm{mg} / \mathrm{kg}$ and $250 \mathrm{mg} /$ $\mathrm{kg}$ ) were determined following determination of the Lethal Dose 50 in the acute toxicity study. The rats were marked such that first group (control) was unmarked, the second group (150mg/ $\mathrm{kg}$ ) was marked by one tail mark, the third group $(200 \mathrm{mg} / \mathrm{kg})$ by two tail marks and the third group $(250 \mathrm{mg} / \mathrm{kg}$ ) by three tail marks. Body weights were measured and recorded at every time of CASBMCE administration.

\section{Collection of tissue samples}

Blood sample collection: Blood samples were collected on the 28th day (the last day of the experiment) from retro orbital plexus of the eye using VITREX NRIS soda lime glass 80IU/ $\mathrm{ml}$ heparinised microhematocrit tubes into EDTA and plain vacutainer tubes for whole blood and serum samples respectively thereafter rats were sacrificed and vital organs (liver, lung, kidney and spleen) collected and fixed in $10 \%$ buffered formalin.

Relative organ weights (row) assay: The relative vital organ weights were taken by using Meltzer sensitive weighing balance.

Hematology: Blood samples were analyzed using NS4s automated analyzer (Germany). The hematological parameters analyzed included: Packed cell volume (PCV), Red Blood Cell (RBC) count, White Blood Cell Count (WBCC) and Hemoglobin concentration $(\mathrm{Hb})$. Others were; mean corpuscular volume (MCV), Mean Corpuscular Hemoglobin (MCH) and Mean Corpuscular Hemoglobin Concentration (MCHC).

Biochemical parameters examination: Biochemical parameters were analysed using procedures outlined in the Biosystems laboratory kit (S.A. Costa Brava, 30. 08030, Barcelona, Spain). The serum samples were assayed for lipid profile (cholesterol and triglycerides), liver marker enzymes (ALT, AST and ALP), kidney function tests (serum urea and creatinine), blood glucose, albumin, total protein and bilirubin.

Histopathology: At the end of the experiments all rats 


\section{Journal of Complementary Medicine \& Alternative Healthcare}

were humanely sacrificed, vital organs were taken and fixed in buffered formalin $10 \%$, for one week, then washed in ascending grades of ethanol, cleared with xylene, embedded in paraffin wax, sectioned by microtome and stained with Haematoxylin and Eosin (H \& E) then mounted on Canada balsam. All sections were examined under a light microscope at 4x,10x, 20x 40x and $100 \mathrm{x}$ magnifications and Photomicrographs taken using Olympus photomicroscope for observation and documentation of histopathological lesions.

Statistical data analysis: Results are presented as mean \pm SEM and were analyzed using One Way-ANOVA (Statistica 8). The means differences were tested using Post Hoc, Fisher LSD and values of $\mathrm{P}<0.05$ were considered statistically significant.

\section{Results}

\section{Acute toxicity}

Just after CASBMCE administration, mice showed sign of rough hair, dyspnoea, sleep, eyelid closure. Three female mice died at a dose of $1200 \mathrm{mg} / \mathrm{kg}$ in first four hours and on day two and day two deaths was recorded at $600 \mathrm{mg} / \mathrm{kg}$.

Male mortalities were recorded in the first day at a dose of
$1200 \mathrm{mg} / \mathrm{kg}$ where all five mice died. After a week three mice died at a dose of $600 \mathrm{mg} / \mathrm{kg}$. No mortality at $300 \mathrm{mg} / \mathrm{kg}$, the remaining mice resumed to normal until sacrificed. The highest dose that didn't kill the mice was $300 \mathrm{mg} / \mathrm{kg}$ and the minimum dose that killed mice was $600 \mathrm{mg} / \mathrm{kg}$. The Lethal Dose Fifty (LD50) was $424 \mathrm{mg} / \mathrm{kg}$.

\section{Clinical observation in subacute toxicity}

Throughout 28 days of the sub acute toxicity study, no sign of toxicity or mortality were observable.

\section{Body weight in subacute toxicity study}

Body weights of both sexes of rats that received CASBMCE increased from day 0 to day 28. The $(\mathrm{P}<0.05)$ suggesting significant weight increase in each group. At Day 0, for males $(\mathrm{P}>0.05)$ and for females at Day $14(\mathrm{P}>0.05)$ suggesting there was no significant weight increase.

\section{Relative organ weights (ROW) in subacute toxicity study}

ROW of vital organs of tested rats significantly decreased $(\mathrm{P}<0.05)$ however the lungs of male rats significantly increased $(\mathrm{P}<0.05)$ compared to control (Table 1).

Table 1: ROW changes in rats CASBMCE after sub acute exposure expressed as mean \pm sem.

\begin{tabular}{|c|c|c|c|c|c|c|}
\hline \multirow{2}{*}{ Sex } & & \multicolumn{5}{|c|}{ Treatment (Dose in mg/kg) } \\
\cline { 2 - 7 } & Parameter & 0 & 150 & 200 & 250 & P-Values \\
\hline Males & Lungs(g) & $0.82 \pm 0.01^{\mathrm{a}}$ & $0.92 \pm 0.09^{\mathrm{d}}$ & $0.83 \pm 0.01^{\mathrm{b}}$ & $0.85 \pm 0.01^{\mathrm{c}}$ & 0.0037 \\
\hline & Kidney(g) & $0.99 \pm 0.02^{\mathrm{a}}$ & $0.97 \pm 0.01^{\mathrm{a}}$ & $0.8 \pm 0.02^{\mathrm{c}}$ & $0.72 \pm 0.03^{\mathrm{b}}$ & 0 \\
\hline & Liver(g) & $3.74 \pm 0.02^{\mathrm{d}}$ & $3.68 \pm 0.01^{\mathrm{c}}$ & $3.66 \pm 0.01^{\mathrm{b}}$ & $3.22 \pm 0.11^{\mathrm{a}}$ & 0.0009 \\
\hline & Heart(g) & $0.66 \pm 0.01^{\mathrm{d}}$ & $0.59 \pm 0.01^{\mathrm{c}}$ & $0.53 \pm 0.01^{\mathrm{b}}$ & $0.46 \pm 0.02^{\mathrm{a}}$ & 0 \\
\hline & Spleen(g) & $0.35 \pm 0.01^{\mathrm{c}}$ & $0.31 \pm 0.01^{\mathrm{a}}$ & $0.29 \pm 0.01^{\mathrm{a}}$ & $0.25 \pm 0.01 \mathrm{~b}$ & 0.00003 \\
\hline & Lung(g) & $1.5 \pm 0.01^{\mathrm{d}}$ & $1.28 \pm 0.05 \mathrm{c}$ & $0.92 \pm 0.02^{\mathrm{b}}$ & $0.59 \pm 0.07^{\mathrm{a}}$ & 0.0005 \\
\hline & Kidney(g) & $1.25 \pm 0.02^{\mathrm{d}}$ & $1.07 \pm 0.04 \mathrm{c}$ & $0.99 \pm 0.03^{\mathrm{b}}$ & $0.84 \pm 0.05^{\mathrm{a}}$ & 0 \\
\hline & Liver(g) & $6.04 \pm 0.02^{\mathrm{d}}$ & $4.83 \pm 0.27 \mathrm{c}$ & $3.35 \pm 0.06^{\mathrm{b}}$ & $3.07 \pm 0.06^{\mathrm{a}}$ & 0.0007 \\
\hline & Heart(g) & $0.6 \pm 0.04^{\mathrm{b}}$ & $0.59 \pm 0.03^{\mathrm{b}}$ & $0.57 \pm 0.02^{\mathrm{b}}$ & $0.49 \pm 0.03^{\mathrm{a}}$ & 0.0034 \\
\hline & Spleen(g) & $0.53 \pm 0.02^{\mathrm{c}}$ & $0.4 \pm 0.03^{\mathrm{b}}$ & $0.29 \pm 0.01^{\mathrm{a}}$ & $0.3 \pm 0.02^{\mathrm{a}}$ & 0 \\
\hline
\end{tabular}

\section{Hematology in subacute toxicity study}

Table 2: ROW changes in rats CASBMCE after sub acute exposure expressed as mean \pm sem.

\begin{tabular}{|c|c|c|c|c|c|c|}
\hline \multirow{2}{*}{ Sex } & & \multicolumn{5}{|c|}{ Treatment (Dose in $\mathrm{mg} / \mathrm{kg})$} \\
\cline { 2 - 7 } & Parameter & 0 & 150 & 200 & 250 & P-Values \\
\hline Males & WBC & $127.75 \pm 0.42^{\mathrm{c}}$ & $103 \pm 6.01^{\mathrm{a}}$ & $111.23 \pm 0.36^{\mathrm{b}}$ & $161.25 \pm 12.16^{\mathrm{d}}$ & 0.0005 \\
\hline & LYMP & $60.96 \pm 0.2^{\mathrm{c}}$ & $45.84 \pm 3.7^{\mathrm{a}}$ & $55.26 \pm 0.18^{\mathrm{b}}$ & $99 \pm 10.77^{\mathrm{d}}$ & 0.0005 \\
\hline & MON & $17.72 \pm 0.06^{\mathrm{a}}$ & $23.42 \pm 1.41^{\mathrm{b}}$ & $25.86 \pm 0.24^{\mathrm{c}}$ & $27.93 \pm 0.43^{\mathrm{d}}$ & 0.0005 \\
\hline & NEU & $49.05 \pm 0.16^{\mathrm{d}}$ & $30.12 \pm 4.62^{\mathrm{a}}$ & $33.73 \pm 0.11^{\mathrm{b}}$ & $34.42 \pm 0.42^{\mathrm{c}}$ & 0.0001 \\
\hline & WBC & $20.03 \pm 0.09^{\mathrm{a}}$ & $30.43 \pm 13.61^{\mathrm{b}}$ & $91.09 \pm 0.3^{\mathrm{c}}$ & $105.73 \pm 3.67^{\mathrm{d}}$ & 0.0005 \\
\hline & LYMP & $8.01 \pm 0.03^{\mathrm{a}}$ & $22.74 \pm 10.17^{\mathrm{b}}$ & $58.66 \pm 0.19^{\mathrm{c}}$ & $58.86 \pm 0.21^{\mathrm{d}}$ & 0.001 \\
\hline & MON & $4 \pm 0.01^{\mathrm{a}}$ & $6.03 \pm 2.7^{\mathrm{b}}$ & $20.02 \pm 0.07^{\mathrm{c}}$ & $29.63 \pm 2.37^{\mathrm{d}}$ & 0.0005 \\
\hline & NEU & $8.02 \pm 0.05^{\mathrm{a}}$ & $1.66 \pm 0.74^{\mathrm{b}}$ & $12.41 \pm 0.04^{\mathrm{c}}$ & $17.24 \pm 1.2^{\mathrm{d}}$ & 0.0005 \\
\hline
\end{tabular}




\section{Journal of Complementary Medicine \& Alternative Healthcare}

Males rats exposed to CASBMCE at doses $(150,200$ and $250 \mathrm{mg} / \mathrm{kg}$ ) of CASBMCE showed significant increase in WBCC $(\mathrm{P}<0.05)$ at dose $250 \mathrm{mg} / \mathrm{kg}$, that is reflected with increase of lymphocytes and monocytes with significant decrease in neutrophils. At a dose of $150 \mathrm{mg} / \mathrm{kg}$ and $200 \mathrm{mg} / \mathrm{kg}$, WBCC decreased significantly compared to control. In females WBCC increased significantly $(\mathrm{P}<0.05)$ and was reflected in lymphocytes, monocytes and neutrophils in all doses to control groups (Table 2).

Table 3: Effects of CASBMCE on male RBC counts and its indices after subacute exposure expressed as mean \pm sem.

\begin{tabular}{|c|c|c|c|c|c|c|}
\hline \multirow{2}{*}{ Sex } & & \multicolumn{5}{|c|}{ Treatment (Dose in mg/kg) } \\
\cline { 2 - 7 } & Parameter & 0 & 150 & 200 & 250 & P-Values \\
\hline Males & RBC & $16.41 \pm 0.05^{\mathrm{d}}$ & $11.95 \pm 1.08^{\mathrm{c}}$ & $8.29 \pm 0.35^{\mathrm{b}}$ & $7.34 \pm 0.3^{\mathrm{a}}$ & 0.0005 \\
\hline & MCV & $55.96 \pm 0.18^{\mathrm{a}}$ & $56.62 \pm 0.24^{\mathrm{b}}$ & $60.92 \pm 0.81^{\mathrm{d}}$ & $58.34 \pm 0.89^{\mathrm{c}}$ & 0.0004 \\
\hline & HCT & $91.69 \pm 0.3^{\mathrm{d}}$ & $67.67 \pm 5.84^{\mathrm{c}}$ & $50.38 \pm 1.47^{\mathrm{b}}$ & $42.84 \pm 1.97^{\mathrm{a}}$ & 0.0005 \\
\hline & MCH & $10.31 \pm 0.03^{\mathrm{a}}$ & $11.01 \pm 0.18^{\mathrm{b}}$ & $19.58 \pm 0.79^{\mathrm{c}}$ & $20.12 \pm 0.52^{\mathrm{d}}$ & 0.001 \\
\hline & MCHC & $18.42 \pm 0.06^{\mathrm{a}}$ & $19.45 \pm 0.27^{\mathrm{b}}$ & $32.09 \pm 0.85^{\mathrm{c}}$ & $34.46 \pm 0.74^{\mathrm{d}}$ & 0.0005 \\
\hline & RDW & $19.82 \pm 0.06^{\mathrm{b}}$ & $11.64 \pm 2.14^{\mathrm{a}}$ & $19.91 \pm 0.54^{\mathrm{c}}$ & $25.88 \pm 1.63^{\mathrm{d}}$ & 0.0011 \\
\hline & HB & $16.92 \pm 0.06^{\mathrm{d}}$ & $16.16 \pm 0.19^{\mathrm{c}}$ & $14.81 \pm 0.05 \mathrm{~b}^{\mathrm{b}}$ & $13.21 \pm 0.39^{\mathrm{a}}$ & 0.0005 \\
\hline & RBC & $5.27 \pm 0.02^{\mathrm{a}}$ & $5.19 \pm 0.02^{\mathrm{b}}$ & $10.86 \pm 0.04^{\mathrm{c}}$ & $16.53 \pm 2.32^{\mathrm{d}}$ & 0.0006 \\
\hline & MCV & $59.26 \pm 0.19^{\mathrm{b}}$ & $64.48 \pm 1.31^{\mathrm{d}}$ & $59.65 \pm 0.03^{\mathrm{c}}$ & $58.15 \pm 1.28^{\mathrm{a}}$ & 0.0008 \\
\hline & HCT & $31.13 \pm 0.1^{\mathrm{a}}$ & $33.43 \pm 0.59^{\mathrm{b}}$ & $64.76 \pm 0.21^{\mathrm{c}}$ & $96.1 \pm 12.6^{\mathrm{d}}$ & 0.0005 \\
\hline & MCH & $23.02 \pm 0.07^{\mathrm{c}}$ & $31.47 \pm 2.14^{\mathrm{d}}$ & $11.82 \pm 0.04^{\mathrm{b}}$ & $7.99 \pm 2.52^{\mathrm{a}}$ & 0.0005 \\
\hline & MCHC & $38.94 \pm 0.13^{\mathrm{d}}$ & $38.34 \pm 0.17^{\mathrm{c}}$ & $19.81 \pm 0.07^{\mathrm{b}}$ & $13.41 \pm 5.62^{\mathrm{a}}$ & 0.0005 \\
\hline & RDW & $11.71 \pm 0.04^{\mathrm{a}}$ & $10.81 \pm 0.22^{\mathrm{b}}$ & $12.16 \pm 0.04 \mathrm{c}$ & $13.51 \pm 0.31^{\mathrm{d}}$ & 0.0005 \\
\hline & HB & $12.11 \pm 0.04^{\mathrm{a}}$ & $12.8 \pm 0.17^{\mathrm{b}}$ & $12.83 \pm 0.03 \mathrm{c}$ & $12.86 \pm 0.04^{\mathrm{d}}$ & 0.0094 \\
\hline
\end{tabular}

RBC, hematocrit, hemoglobin concentration in males significantly decreased at all doses $(\mathrm{P}<0.05)$ compared to control. Hematological indices $\mathrm{MCV}, \mathrm{MCH}$ and $[\mathrm{MCHC}$ ] were significantly increased $(\mathrm{P}<0.05)$ compared to control. The RDW significantly decreased at $150 \mathrm{mg} / \mathrm{kg}$ and significantly increased at $200 \mathrm{mg} / \mathrm{kg}$ and $250 \mathrm{mg} / \mathrm{kg}$ in both sexes compared to control group (Table $3)$.

Table 4: Effects of subacute exposure of CASBMCE on rats biochemical parameters expressed as mean \pm sem.

\begin{tabular}{|c|c|c|c|c|c|c|}
\hline \multirow{2}{*}{ Sex } & \multirow{2}{*}{ Parameter } & \multicolumn{5}{|c|}{ Treatment (Dose in mg/kg) } \\
\hline & & 0 & 150 & 200 & 250 & P-Values \\
\hline \multirow{8}{*}{ Males } & Glucose(mg/dl) & $33.9 \pm 0.03^{\mathrm{a}}$ & $68.46 \pm 8.49^{b}$ & $72.45 \pm 0.07^{c}$ & $81.76 \pm 2.32^{\mathrm{d}}$ & 0.0005 urea \\
\hline & Cholesterol(mg/dl) & $129.96 \pm 0.12^{\mathrm{d}}$ & $70.17 \pm 14.6^{c}$ & $62.86 \pm 0.06^{\mathrm{b}}$ & $48.96 \pm 3.37^{\mathrm{a}}$ & 0.0005 \\
\hline & Total Protein (g/dl) & $8.44 \pm 0.01^{d}$ & $8.28 \pm 0.03^{c}$ & $7.57 \pm 0.01^{b}$ & $6.31 \pm 0.3^{\mathrm{a}}$ & 0.0005 \\
\hline & Albumin(g/dl) & $3.36 \pm 0^{c}$ & $3.38 \pm 0.01^{\mathrm{d}}$ & $2.64 \pm 0.24^{\mathrm{b}}$ & $2.49 \pm 0.16^{\mathrm{a}}$ & 0.0011 \\
\hline & Triglycerides(mg/d) & $39.63 \pm 0.04^{\mathrm{a}}$ & $115.99 \pm 18.74^{\mathrm{d}}$ & $114.82 \pm 0.1^{\mathrm{c}}$ & $69.59 \pm 11.07^{b}$ & 0.0005 \\
\hline & Urea(mg/dl) & $29.01 \pm 0.06^{\mathrm{d}}$ & $24.34 \pm 1.05^{c}$ & $16.36 \pm 0.02^{b}$ & $13.58 \pm 0.69^{a}$ & 0.0005 \\
\hline & Bilirubin (mg/dl) & $0.67 \pm 0.01^{d}$ & $0.5 \pm 0.03^{\mathrm{c}}$ & $0.85 \pm 0.01^{b}$ & $1.06 \pm 0.06^{\mathrm{a}}$ & 0 \\
\hline & Creatinine(mg/dl) & $0.74 \pm 0.01^{\mathrm{d}}$ & $0.67 \pm 0.03^{c}$ & $0.89 \pm 0.01^{b}$ & $1.03 \pm 0.05^{\mathrm{a}}$ & 0 \\
\hline
\end{tabular}

\section{Biochemical assays in subacute toxicity}

The 28 day repeated dose administration produced changes in blood serum parameters in dose related fashion.

\section{Glucose level in subacute toxicity study}

Exposure of rats caused increase in blood glucose in male and female rats administered with CASBMCE compared to control group $(\mathrm{P}<0.05)$ in both sexes (Table 4$)$. 
Journal of Complementary Medicine \& Alternative Healthcare

\begin{tabular}{|c|c|c|c|c|c|c|}
\hline \multirow{5}{*}{ Females } & Glucose $(\mathrm{mg} / \mathrm{dl})$ & $64.48 \pm 0.25^{\mathrm{a}}$ & $77.44 \pm 3.28^{\mathrm{b}}$ & $97.71 \pm 0.09^{\mathrm{c}}$ & $98.04 \pm 0.16^{\mathrm{d}}$ & 0.0007 \\
\cline { 2 - 7 } & Cholesterol(mg/dl) & $129.1 \pm 0.5^{\mathrm{d}}$ & $59.24 \pm 16.92^{\mathrm{c}}$ & $56.21 \pm 0.05^{\mathrm{b}}$ & $41.1 \pm 3.67^{\mathrm{a}}$ & 0.0005 \\
\cline { 2 - 7 } & Total Protein (g/dl) & $6.5 \pm 0.02^{\mathrm{a}}$ & $7.85 \pm 0.34^{\mathrm{c}}$ & $7.09 \pm 0.01^{\mathrm{b}}$ & $8.15 \pm 0.26^{\mathrm{d}}$ & 0.0005 \\
\cline { 2 - 7 } & Albumin(g/dl) & $2.55 \pm 0.04^{\mathrm{a}}$ & $3.13 \pm 0.16 \mathrm{~d}$ & $3.03 \pm 0.05 \mathrm{c}$ & $2.76 \pm 0.06 \mathrm{~b}$ & 0.0008 \\
\cline { 2 - 7 } & Triglycerides(mg/dl) & $81.91 \pm 2.31^{\mathrm{c}}$ & $132.42 \pm 13.07^{\mathrm{d}}$ & $70.56 \pm 0.07^{\mathrm{b}}$ & $39.63 \pm 7.54^{\mathrm{a}}$ & 0.0005 \\
\cline { 2 - 7 } & Urea(mg/dl) & $48.51 \pm 0.05^{\mathrm{d}}$ & $27.97 \pm 5.01 \mathrm{c}$ & $24.84 \pm 0.02^{\mathrm{b}}$ & $18.22 \pm 1.61^{\mathrm{a}}$ & 0.0005 \\
\hline \multirow{3}{*}{} & Bilirubin (mg/dl) & $0.35 \pm 0.12^{\mathrm{a}}$ & $0.5 \pm 0.41^{\mathrm{b}}$ & $0.8 \pm 0.01^{\mathrm{c}}$ & $1.61 \pm 0.21^{\mathrm{d}}$ & 0.0006 \\
\hline & Creatinine (mg/dl) & $0.82 \pm 0.01^{\mathrm{d}}$ & $0.44 \pm 0.19^{\mathrm{a}}$ & $0.74 \pm 0.03^{\mathrm{b}}$ & $0.81 \pm 0.03^{\mathrm{c}}$ & 0.0012 \\
\hline
\end{tabular}

\section{Lipid profile in subacute toxicity study}

6.8.1.Cholesterol: The exposure of rats to CASBMCE caused significant decrease $(\mathrm{P}<0.05)$ of cholesterol level in both sexes compared to control groups (Table 4).

6.8.2.Triglyceride: The results show that in males there is significant increase $(\mathrm{P}<0.05)$ in triglycerides levels in test group compared to control group, at $150 \mathrm{mg} / \mathrm{kg}$ there was more increase than at higher dose. Females show that there is significant increase of serum triglyceride at $150 \mathrm{mg} / \mathrm{kg}$ and significant decrease at dose $200 \mathrm{mg} / \mathrm{kg}$ and $250 \mathrm{mg} / \mathrm{kg}(\mathrm{P}<0.05)$ as compared to control group (Table 4).

\section{Total protein in subacute toxicity study}

There is significant decrease of total protein in males $(\mathrm{P}<0.05)$ and significant increase in female serum total protein $(\mathrm{P}<0.05)$ compared control groups (Table 4$)$.

\section{Total bilirubin in subacute toxicity study}

Bilirubin significantly increased in both sexes $(\mathrm{P}<0.005)$ compared to control group and significant decrease at $150 \mathrm{mg} /$ $\mathrm{kg}$ in males compared to control (Table 4).

\section{Kidney function in subacute toxicity study}

Serum urea: Significant decrease in blood urea was recorded in treated rats $(\mathrm{P}<0.05)$ as compared to control groups (Table 4$)$.

Serum creatinine: Males serum creatinine significantly increased $(\mathrm{P}<0.05)$ at a dose of $200 \mathrm{mg} / \mathrm{kg}$ and at $250 \mathrm{mg} / \mathrm{kg}$. At a dose of $150 \mathrm{mg} / \mathrm{kg}$ in males and all doses for females creatinine decreased significantly $(\mathrm{P}<0.05)$ compared to control (Table 4$)$.

Serum albumin: Albumin significantly increased $(\mathrm{P}<0.05)$ in female rats. In males albumin significantly increased $(\mathrm{P}<0.05)$ at $150 \mathrm{mg} / \mathrm{kg}$ and decreased at $200 \mathrm{mg} / \mathrm{kg}$ and $250 \mathrm{mg} / \mathrm{kg}(\mathrm{P}<0.05)$ compared to control group (Table 4).

\section{Liver Enzyme Markers}

\section{Alanine amino transferase (ALT)}

Table 5: Effects of CASBMCE on liver enzymes of rats after subacute exposure expressed as mean \pm sem.

\begin{tabular}{|c|c|c|c|c|c|c|}
\hline \multirow{3}{*}{ Sex } & \multirow{3}{*}{ Parameter } & \multicolumn{5}{|c|}{ Treatment (Dose in mg/kg) } \\
\cline { 3 - 7 } & & 0 & 150 & 200 & 250 & \multicolumn{2}{c|}{ P-Values } \\
\hline \multirow{3}{*}{ Males } & ALT(U/L) & $27.26 \pm 0.56^{\mathrm{a}}$ & $29.93 \pm 1.11^{\mathrm{b}}$ & $39.91 \pm 0.67^{\mathrm{c}}$ & $46.56 \pm 2.22^{\mathrm{d}}$ & 0.0005 \\
\cline { 2 - 7 } & AST(U/L) & $58.19 \pm 1.06^{\mathrm{a}}$ & $59.89 \pm 1.36^{\mathrm{a}}$ & $69.84 \pm 1.17^{\mathrm{b}}$ & $76.49 \pm 2.67^{\mathrm{c}}$ & 0 \\
\cline { 2 - 8 } & ALP(U/L) & $54.37 \pm 0.69^{\mathrm{d}}$ & $53.92 \pm 0.78^{\mathrm{c}}$ & $42.47 \pm 0.71^{\mathrm{b}}$ & $35.02 \pm 1.4 \mathrm{a}$ & 0.0011 \\
\hline \multirow{3}{*}{ Females } & ALT(U/L) & $16.67 \pm 0.06^{\mathrm{a}}$ & $19.95 \pm 0.95^{\mathrm{b}}$ & $33.26 \pm 0.56^{\mathrm{c}}$ & $36.58 \pm 1.31^{\mathrm{d}}$ & 0.0005 \\
\cline { 2 - 8 } & AST(U/L) & $49.84 \pm 1.17^{\mathrm{d}}$ & $49.88 \pm 1^{\mathrm{c}}$ & $63.19 \pm 1.06^{\mathrm{b}}$ & $68.51 \pm 1.78^{\mathrm{a}}$ & 0.0013 \\
\cline { 2 - 8 } & ALP(U/L) & $85.73 \pm 0.33^{\mathrm{a}}$ & $74.7 \pm 2.32^{\mathrm{b}}$ & $69.22 \pm 2.6 \mathrm{c}$ & $66.74 \pm 0.8^{\mathrm{d}}$ & 0.0017 \\
\hline
\end{tabular}

ALT significantly increased $(\mathrm{P}<0.05)$ in both sexes as compared to control (Table 5).

\section{Aspartate amino transferase (AST)}

AST $(\mathrm{P}<0.05)$ significantly increased in both sexes of tested rats as compared to control group (Table 5).

\section{Alkaline phosphatase (ALP)}

ALP $(\mathrm{P}<0.05)$, in both sexes decreased significantly compared to control group (Table 5). 


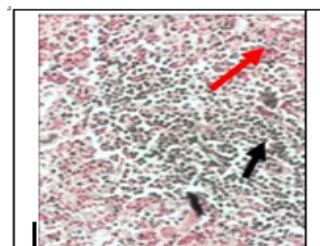

Figure 1:

Female control spleen

administered

with DMSO

showing normal distribution red pulp (red arrow) and white pulp

(black arrow)

(H\&E stain

magnification $40 \mathrm{x}$ )

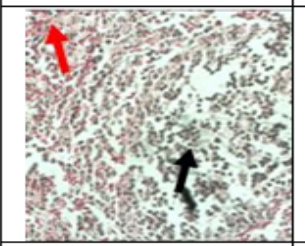

Figure 7: Spleen of male rat administered with CASBMCE $200 \mathrm{mg} / \mathrm{kg}$ showing red pulp (red arrow) and white pulp (black arrow) (H\&E stain magnification 40x)

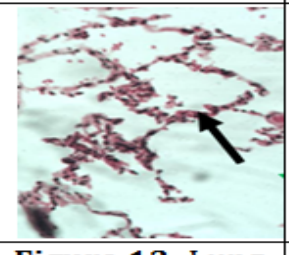

Figure 13: Lung of male control rat administered with DMSO showing normal alveolar wall (black arrow) of the lung. (H\&E stain magnification 40x)

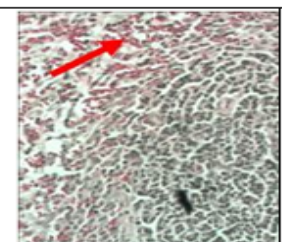

Figure 2:

Female rat spleen administered with $150 \mathrm{mg} / \mathrm{kg}$ CASBMCE showing red pulp (red arrow) and white pulp

(black arrow)

(H\&E stain magnification 40x)

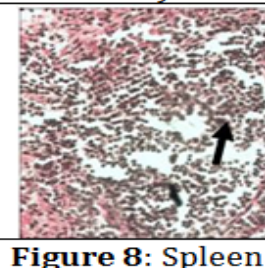

Figure 8: Splee administered with CASBMCE $250 \mathrm{mg} / \mathrm{kg}$ showing red pulp (red arrow) and white pulp(black arrow). (H\&E stain magnification 40x)

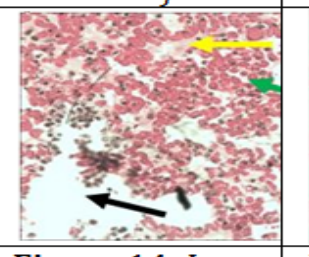

Figure 14: Lung

of the male rat administered with CASBMCE at $150 \mathrm{mg} / \mathrm{kg}$ showing scattered RBC(green arrow),alveolar sac (black arrow) and alveolar wall(yellow arrow) (H\&E stain magnification

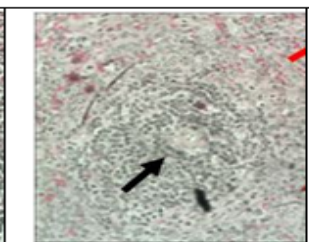

Figure 3: Spleen of female rat administered with $200 \mathrm{mg} / \mathrm{kg}$ CASBMCE

showing reduced red pulp and white pulp (atrophy).

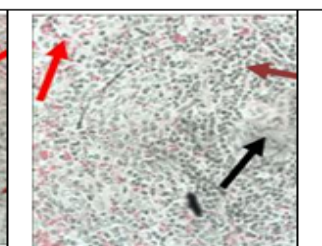

Figure 4: Spleen of a female rat administered with $250 \mathrm{mg} / \mathrm{kg}$ CASBMCE showing more reduced red pulp and white pulp (atrophy).

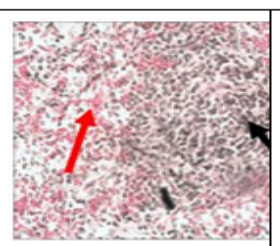

Figure 5:

Spleen of male

control rat

administered

with DMSO

showing normal

spleen red pulp

(red arrow) and

white pulp

(black arrow)

(H\&E stain

magnification of 40x)

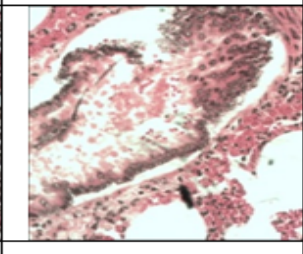

Figure 9: Lung

of the female control rat after administration of DMSO

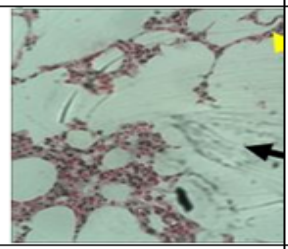

\section{Figure 15: Lung}

of male rat

administered with CASBMCE at $200 \mathrm{mg} / \mathrm{kg}$ showing normal alveolar wall (yellow arrow) and alveolar sac (black arrow) of the lung. (H\&E stain magnification $40 \mathrm{x}$

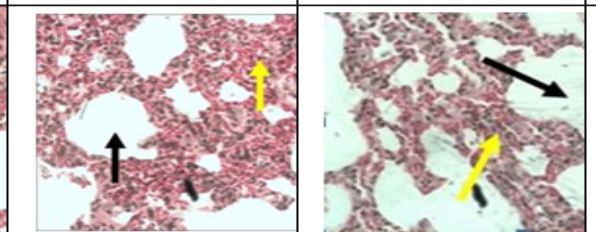

Figure 10: Lung of the female rat given a dose of $150 \mathrm{mg} / \mathrm{kg}$ CASBMCE showing thickened alveolar walls (yellow arrow) and surrounded alveolar sac (black arrow).

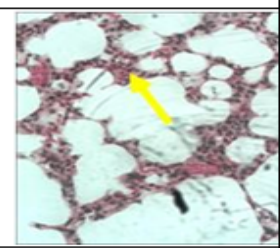

Figure 16: Lung of the male rat administered with CASBMCE at $250 \mathrm{mg} / \mathrm{kg}$ showing normal alveolar wall(yellow arrow) and alveolar sac(black arrow).(H\&E stain magnification 40x)
Figure 11: Lung of female rat given CASBMCE at $200 \mathrm{mg} / \mathrm{kg}$ showing thickened alveolar walls (yellow arrow) and respiratory tract (black arrow).

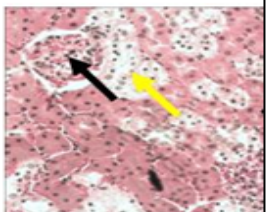

Figure 17:

Kidney of control female rat

administered with DMSO showing normal proximal convoluted tubules (yellow arrow) and glomeruli(black arrow) (H\&E stain $40 \mathrm{x}$ ) magnification

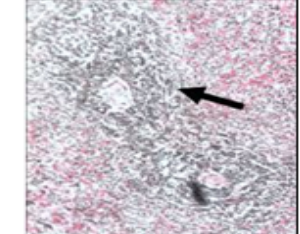

Figure 6: Spleen of male rat administered with CASBMCE $150 \mathrm{mg} / \mathrm{kg}$ showing red pulp (red arrow) and white pulp

(black arrow)

(H\&E stain magnification 40x)

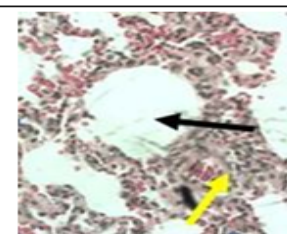

Figure 12: lung of female rats given CASBMCE at a dose of $250 \mathrm{mg} / \mathrm{kg}$ showing thickened alveolar wall (yellow arrow) and

circumscribed alveolar sac (black arrow)

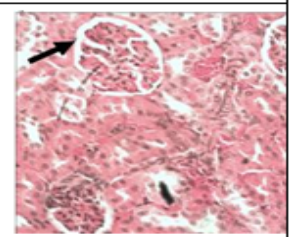

Figure 18:

Kidney of a

female rat

administered with CASBMCE at

$150 \mathrm{mg} / \mathrm{kg}$ showing widened glomeruli space(black arrow) (H \& E stain

magnification $40 \mathrm{x}$ ) $40 \mathrm{x}$ ) 


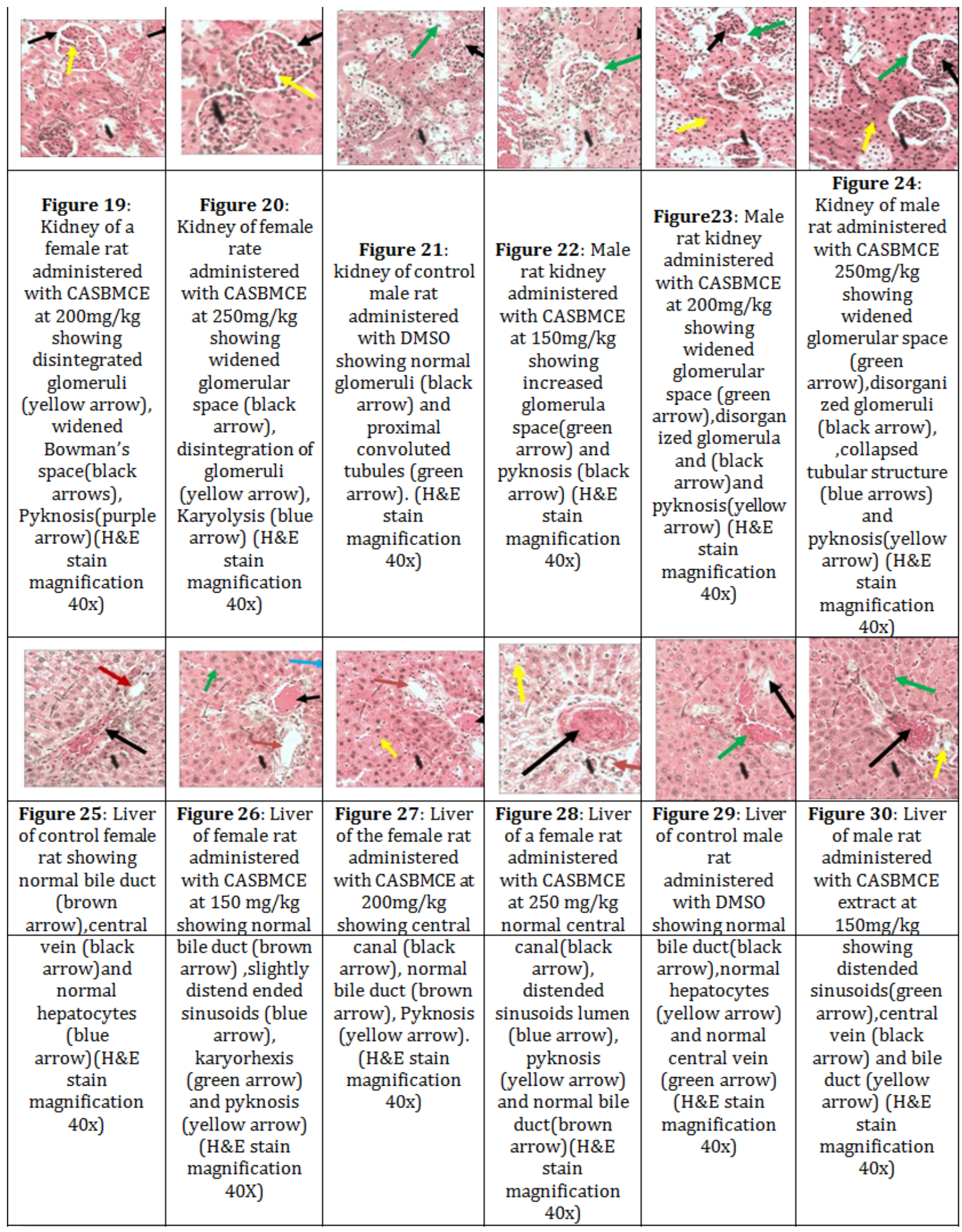




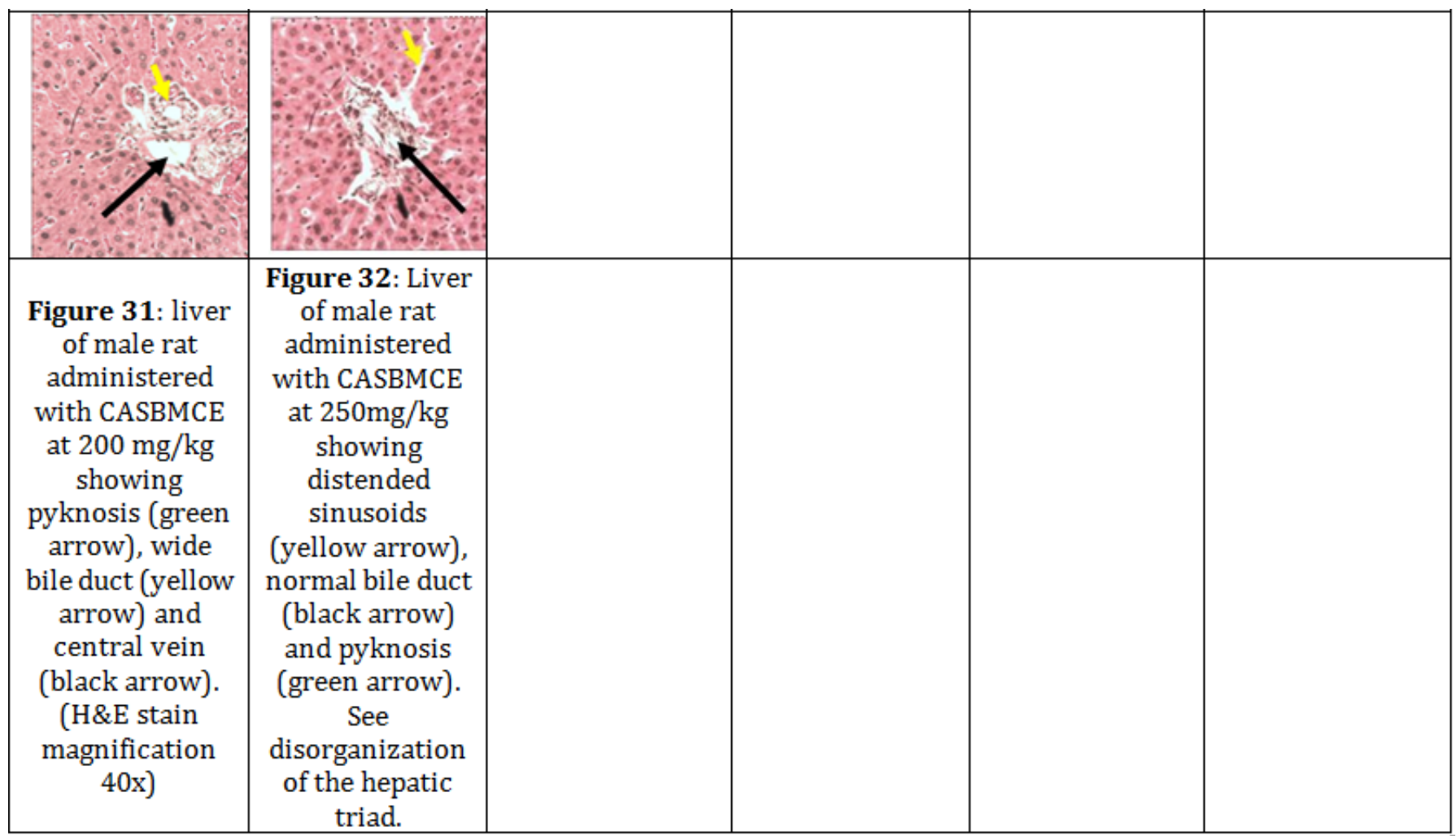

Oral administration of the CASBMCE at different doses showed different pathological lesions in vital organs on micrographs. The spleen show normal red and white pulp in females (Figure 1-4) and male spleen (Figure 5-8). Female lungs alveolar walls are thickened with narrowed respiratory ducts (Figure 9-12), where males and control groups appears normal (Figure 13-16). Kidneys in both sexes shows increased glomerular space with lost structure, tubular cells are pyknotic in female (Figure 17-20) and male kidney (Figure 21-24). Liver triads appear normal with pyknosis in female (Figure 25-28) and Male liver hepatocytes (Figure 29-32).

\section{Discussion}

Herbal medicines have been used extensively in many African countries including Tanzania as an alternative remedy for different diseases; however their toxicological profiles have not been studied to validate their effects to body tissues. For instance some herbal medicines were made from aristoholic acid led to renal failure, WHO makes emphasis on herbal medicines quality [8]. Commiphora species are used in different humans' ailments management with different side effects [9]. C. campestris have been used as medication of various ailments by Pare people since long time, without guarantee by any toxilogical studies. The plant roots, stem, bark and leaf were tested for antibacterial effects and showed to be effective against several gram positive and gram negative organisms [10].

Acute toxicity study mortalities at a dose of $600 \mathrm{mg} / \mathrm{kg}$ and $1200 \mathrm{mg} / \mathrm{kg}$ shows that at high dose CASBMCE is toxic but is safe in lower doses. Increase in body weight reveal that the CASBMCE had no negative impact on the growth.
Relative Organ Weight (ROW) of kidney, liver, heart and spleen were significantly reduced in test rats as compared to control group. CASBMCE had effects on the organ weight during sub acute exposure. Kidney shrinkage was observed with increased glomerular space and might have led to reduced renal function hence increased blood creatinine and reduced erythrocytes production. The kidney is a good source of erythropoietin hormone essential for stimulation of erythrocytes production from hemopoietic organs, shrinkage might have altered reduced the hormone quantity resulting to low RBC production in the male rats (Table 3). Reduced liver size (Table 5) might have led to increased levels of AST, ALT and increased urea levels (Table 5). Impaired deamination might have caused low urea production (Table 4 \& 5) [8]. The spleen is involved in production of RBC, destruction of damaged RBC and lymphocytes [11]. CASBMCE might be directly associated with spleen atrophy (decreased spleen size) [12], that caused reduction in circulating RBC seen in male rats, because spleen plays a role to control the amount of RBC in circulation. Spleen micrographs (Figure 17-20) show splenic extramedulary hemopoiesis to try to cover the decreased amount of RBC in circulation [13]. Female rats showed increased RBC levels with increasing doses, this indicates that there was no effect on the hematopoietic organs. However, reduced spleen weight might have contributed to decreased hemoglobin levels (Figure 1-4), due to spleen failure to maintain RBC in circulation [13]. The RDW was high in females, this means the RBC had varied shape compared to normal cells, this also is a factor for low (Hb) although RBC numbers are high.

Increased total WBCC, lymphocytes and monocytes, this suggests that CASBMCE didn't affect the WBC haemopoietic sites. 
Studies show that anticancer chemotherapies have a tendency to raise lymphocyte numbers by producing interleukine 2 that modulates cytokines that promote differentiation of lymphocyte precursor cells into mature lymphocytes $[14,15]$. Studies with Commiphora molmol (myrrh) have shown that the plant has anticancer effects that could trigger rise of WBC [16], this is corroborated by other studies of C. molmol on WBC lines that have shown that, the species produces cytokines that trigger proliferation of lymphocytes [17], this support the theory that C. campestris might have compounds that are likely to possess anticancer effects similar to those of C. molmol (myrrh). Further studies are needed to validate this.

The RBC counts decreased with dose in males and increased in females, this may be linked to interference of CASBMCE in hematopoietic organs functioning like spleen, liver and kidney RDW is erythrocyte size variability caused by nutrition, heart diseases, iron deficiency and chemotherapy, it has been used as a good indicator of renal disease [18], kidney micrographs (Figure 25-28) show pathological changes that are linked to RDW increase, in this study there was increase in RDW at dose of $250 \mathrm{mg} / \mathrm{kg}$, however, at the dose of 150 and $200 \mathrm{mg} / \mathrm{kg}$, the RDW was lower compared to control, this corroborates with the study that was done in humans that showed increase in RDW associated with renal impairment [19]. RDW means abnormal $\mathrm{RBC}$, this is a feature for anemia seen in female rats. In liver damage cases, RDW relates inversely to albumin, however when there is increased MCV and RDW also defines liver insufficiency [20], these changes signify that CASBMCE caused liver damage.

RBC and hematocrit decrease in males, indicates that CASBMCE interferes with production of RBC in hemopoietic organs. Kidneys produces erythropoietin hormone used in RBC production in the bone marrow [21]. Kidney pathological lesions caused by CASBMCE like destructed renal tubules cells might have impaired kidney erythropoietin production hence anemia, this is supported by studies on human with renal impairment suffered anemia [22].

Increase RBC counts in females and Hematocrit in dose increasing order, meaning there is no effect on erythropoietic processes, however there was slight hemoglobin increase in between doses irrespective of the increased RBC and Hemoglobin, this means may be the liver synthesis mechanism of the protein responsible for hemoglobin might have been affected that led to impaired hemoglobin synthesis, however increased RBC counts could also be a result of reduced oxygen supply in alveoli (hypoalveolar oxygen) in the lungs causing the body to react by producing more $\mathrm{RBC}$ to compensate for oxygen demand [21], this is supported by lung micrograph of the female that shows thickening alveolar walls which reduced oxygen diffusion into body tissues from the lungs hence triggering the effect of enhanced RBC production so as to cover reduced oxygen levels (hypoxia) (Figure 5-8). In males the lungs appeared with normal sized alveolar walls so oxygen diffused normally in the lungs

\section{(Figure 21-24)}

Triglycerides and Cholesterol make up the body lipids, in this study CASBMCE decreased cholesterol and triglycerides compared to controls, so the plant have hypolipidemic effects. In other studies of Commiphora species have shown antilipidemic effects in animals of both sexes, as supported by studies on C. mukul [22].

Total protein decrease in tested rats compared to controls, the cause might be due to liver and kidney malfunction due to CASBMCE, leading to hypoprotenemia [4], this is also confirmed by aberrations in the liver and kidney glomeruli interfering protein synthesis and elimination. In females, increased protein was observed. Which is an indication that the organs were not affected however; this could have been influenced by other female physiological processes which influence rise in total protein levels like estrus cycles [23]?

Albumin is a serum protein that is produced from the liver and is excreted through the kidneys [24]. In this study, decrease of albumin can directly be linked to liver and kidney dysfunction. The effect of CASBMCE in test animals could be a reason for liver failure to synthesize proteins [25]. Liver damage (Figure 13-16, 29-32) and kidney damage (Figure 9-12, 25-28) might have aggravated albumin decrease; this decreased is also seen in hepatitis and liver cirrhosis [4].

Bilirubin is a yellow pigment that is produced after breakdown of the RBC hemoglobin in the liver resulting in protein separation into globin and heme, heme is further broken to biverdin that is reduced into bilirubin, this occurs in the reticuloendothelial system of the liver, spleen and bone marrow [26]. Increased bilirubin is a result of liver or kidney disease. This can also be related to decreased levels of $\mathrm{RBC}$ in males suggesting that there is excessive breaking down of RBC (hemolysis) releasing its hemoglobin that gives rise to total serum bilirubin [4].

There was increase of two liver enzymes ALT and AST in this study. ALT, was increased in levels in treated animals of both sexes compared to control groups, this shows that the CASBMCE has a hepatotoxicity effect, this enzyme however is not only found in the liver alone it could be from other sources in lesser amounts such as kidneys, heart and skeletal muscles. ALT results are still not sufficient to conclude the effects of the plant as it could be originating from other tissues [27], in this study (Figure 13-16, 29-32) liver micrographs shows pyknosis and distended sinusoids suggesting liver damage, hence increase of ALT [28].

AST is found in large amounts in the muscles, liver and heart. AST is found elevated in blood due to damage to cells of muscles, heart muscles and liver and reduced clearance of abnormal compounds in blood plasma and when there is increased hemolysis of RBC [4]. In this study, there is a significant rise in the AST, this may be due to liver damage as it can be supported by micrographs (Figure 14-16, 30-32). 
ALP is an enzyme that catalyses transport of fats in the intestine, however increase in levels of this enzyme in blood is associated with bile duct occlusion or bone diseases, caliculi occlusion or inflammation of bile duct, hence increased need for transmission of the bile [4]. In this study there is significant decrease of ALP, this means the bile ducts were not damaged and this is proven by liver micrographs (Figure 13-16, 29-32) shows normal architecture of the bile ducts. Studies with other species of Genus Commiphora such as C. opobalsamum (syn Balesan) have shown that the species are used in treatment of liver disease and in renal caliculi to facilitate urine excretion [29], so maybe this plant also has the same compounds that could facilitate opening of bile duct resulting in urine excretion, however studies are still needed to validate this.

There was a rise in creatinine levels of test rats compared to the control group. Creatinine is a byproduct of muscle cellular metabolism of creatine for energy production and is removed by kidneys and remains at levels that are fairly reasonable [30]. If creatinine accumulates in the blood it indicates renal impairment, these results show that CASBMCE caused kidney damage, glomerula filtration rate seems to have been affected as creatinine accumulated in blood. In this study all tested rats renal micrographs show that glomerular space has widened and glomerulus is disorganized (Figure 25-28), this could be the reason for insufficiency of creatinine clearance [4].

BUN is blood urea nitrogen; this is formed from breakdown of protein in the gastro intestinal tract content by bacterial protease, urease and amine oxidases. In this study urea was lower in tested rats compared to control group, this may be due to the influence of liver cell damage caused by extracts which led to reduced metabolism of ammonia by hepatocytes resulting in lower urea production. The most common cause of failure to metabolize ammonia is impairment of hepatocytes functioning that is due to liver disease [4]. Lowering of blood urea has been observed in studies with human patients with cirrhosis without azotemia [30].

Glucose increased in the blood of tested rats as compared to control group, suggesting CASBMCE influenced metabolism of carbohydrates into glucose. Glucose level increases are normally observed in animals that are suffering from impairment of pancreas function that leads to production of inadequate or dysfunctional insulin. In this study the CASBMCE could have had an effect on insulin performance, resulting in insulin resistance which reduces the ability of insulin to act on peripheral tissues like skeletal, adipose and liver tissues. However, other studies of Commiphora species such as C. africana have shown that in high dose it reduces blood glucose [31].

\section{Conclusion}

In acute toxicity study CASBMCE, produced mortality in males and females at a dose of $600 \mathrm{mg} / \mathrm{kg}$ and $1200 \mathrm{mg} / \mathrm{kg}$. In sub acute doses CASBMCE, produced no mortality. Major effects were rise of glucose levels, decreased vital organs size, and thickened female lung alveolar walls that probably increased RBC counts. CASBMCE reduced cholesterol levels indicating antilipidemic effect and the Alkaline Phosphatase was decreasing in amount suggesting CASBMCE might have liver repair compounds for bile ducts. More research is needed to profile the chemical compounds in the extract and validation of safety doses in humans.

\section{Acknowledgement}

The authors thank Mr. Alfred Godfrey Mwanyika and Mr. John Mlay (College of Veterinary Medicine and Medical Sciences Morogoro, Sokoine University of Agriculture) for technical advice on Laboratory techniques, blood sample analysis and Microscope tissue glass slides preparation.

\section{Funding}

This study was funded by the TFDA as part of the fee for education carrier development in his staff, but did not take part in any part development of the study design, data collection, manuscript and decision to publish.

\section{References}

1. TFDA (2012) Tanzania food and drugs authority (TFDA), List of registered products, Tanzania government gazette, Tanzania.

2. TFDA (2016) Tanzania food and drugs authority, registered drug products. Tanzania government gazette, Tanzania.

3. WHO (2002) Traditional medicine strategy (2002-2005), Geneva, Switzerland, Europe, pp. 1-59.

4. Godfrey E, Leonard Jones Chauka, Musa Chacha (2015) Antimicrobial and cytotoxicity analysis of selected pare falk in all plants (Dissertation). Spatula DD 5(4): 207-214.

5. Godfrey E (2016) Antimicrobial and cytotoxicity of selected pare falk medicinal plants (A Dissertation submitted in partial fulfillment of the requirements for the degree of masters of life science and bioengineering of the nelson mandela african institution of science and technology), Arusha, Tanzania, pp 21-25.

6. Lorke D (1983) A new aproach to practical acute toxicity testing. Arch Toxicol 54(4): 275-287.

7. OECD (2008) Repeated dose 28-day oral toxicity study in rodents, oecd guidel test chem sect 4, paris. Environment Directorate.

8. WHO (2004) WHO guidelines on safety monitoring of herbal medicines in pharmacovigilance systems. Geneva, pp. 1-47.

9. Paraskeva MP, Vuuren SF Van, Zyl RL Van, Davids H, Viljoen AM, et al. (2008) The in vitro biological activity of selected South African Commiphora species. J Ethnopharmacol 119(3): 673-679.

10. Burtis C, Ashwood ER, Bruns D (2008) In TIETZ Fundamentals of clinical chemistry. (6 $6^{\text {th }}$ edn), Saunders Elsevier 54(11): 976.

11. Ward JM, Rehg JE, Herbert C Morse (2012) Diffentiation of rodent immune and hematopoietic system reactive lesions from neoplasias. Toxicol Pathol 40(3): 724-732.

12. Elmore SA (2006) Enhanced histopathology of the spleen. Toxicol Pathol 34(5): 648-655.

13. Lissoni P, Fumagalli L, Brivio F, Rovelli F, Messina G, et al. (2006) Cancer chemotherapy-induced lymphocytosis: a revolutionary discovery in the medical oncology. J Biol Regul Homeost Agents 20(1-2): 29-35.

14. Lissoni P, Fumagalli L, Paolorossi F, Mandalà M (1999) Changes in lymphocyte number during cancer chemotherapy and their relation to clinical response. Int J Biol Markers 14(2): 115-117. 


\section{Journal of Complementary Medicine \& Alternative Healthcare}

15. Esposito ER, Bystrek MV, Boyle S, Borders J, Willinger L, et al. (2014) Frankincense and Myrrh: A sticky combination for the treatment of lung cancer. Society of toxicology (SOT) $53^{\text {rd }}$ Annual meeting and toxexpo. Phoenix, Arizona 138(1): 78.

16. Haffor A (2010) Effect of Commiphora molmol on leukocytes proliferation in relation to histological alterations before and during healing from injury. Saudi J Biol Sci 17(2): 139-46.

17. Solak Y, Yilmaz MI, Saglam M, Caglar K, Verim S, et al. (2014) Red cell distribution width is independently related to endothelial dysfunction in patients with chronic kidney disease. Am J Med Sci 347(2): 118-124.

18. Tekce H, Kin Tekce B, Aktas G, Tanrisev M, Sit M (2014) The evaluation of red cell distribution width in chronic hemodialysis patients. Int Nephrol doi.org/10.1155/2014/754370.

19. Fisher JW (2003) Erythropoietin: physiology and pharmacology update. Exp Biol Med 228(1): 1-14.

20. Strippoli GFM, Craig JC, Manno C, Schena FP (2004) Hemoglobin targets for the anemia of chronic kidney disease: a meta-analysis of randomized, controlled trials. J Am Soc Nephrol 15(12): 3154-3165.

21. Ritcher T, West J, Alfred Fishman (1957) The syndrome of alveolar hypoventilation and diminished respiratory centre. The New England Journal of Medicine 256: 1165-1170

22. Khanna N, Arora D, Halder S, Mehta AK, Garg GR, et al. (2010) Comparative effect of Ocimum sanctum, Commiphora mukul, folic acid and ramipril on lipid peroxidation in experimentally-induced hyperlipidemia. Indian J Exp Biol 48(3): 299-305.
23. Yaqub Y, Kawu LS, MU, Ayo JO (2013) Influence of reproductive cycle, sex, age and season on haematologic parameters in domestic animals: A review. J Cell Anim Biol 7(4): 37-43.

24. Townsend JC (1990) Increased albumin excretion in diabetes. J Clin Pathol 43(1): 3-8.

25. Thapa BR, Walia A (2007) Liver function tests and their interpretation. Indian J Pediatr 74(7): 663-671.

26. Kakadiya J (2009) Liver function test-a review address for correspondence. Pharmacologyonline 2: 271-282.

27. Ozer J, Ratner M, Shaw M, Bailey W, Schomaker S, et al. (2008) The current state of serum biomarkers of hepatotoxicity. Toxicology 245(3): 194-205.

28. Ezeja MI, Anaga AO, Asuzu IU (2014) Acute and sub-chronic toxicity profile of methanol leaf extract of Gouania longipetala in rats. J Ethnopharmacol 151(3): 1155-1564.

29. Shen T, Li G, Wang X, Lou H (2012) The Genus Commiphora: A review of its traditional uses, phytochemistry and pharmacology. J Ethnopharmacol 142(2): 319-30.

30. Papadakis M, Arieff A (1987) Unpredictability of clinical evaluation of renal function in cirrhosis. prospective study. Am J Med 82(5): 945-952.

31. Goji ADT, Dikko AAU, Bakari AG, Mohammed A, Ezekiel I, et al. (2009) Effect of aqueous-ethanolic stem bark extract of commiphora africana on blood glucose levels on normoglycemic wistar rats. Int J Anim Vet Adv 1(1): 22-24.

\section{Your next submission with Juniper Publishers will reach you the below assets}

This work is licensed under Creative

DOI: 10.19080/JCMAH.2017.04.555629 